\title{
Adherence to Hepatocellular Carcinoma Surveillance and Perceived Barriers Among High-Risk Chronic Liver Disease Patients in Yunnan, China
}

\author{
Jingyi Dai ${ }^{1,2, *}$ \\ Jun Zhao $\mathbb{D}^{3, *}$ \\ Yingrong Du (D) \\ Lin Zhuang' \\ Edward B McNeil (D) ${ }^{2}$ \\ Virasakdi Chongsuvivatwong (D) ${ }^{2}$ \\ 'Department of Liver Diseases, The \\ Third People's Hospital of Kunming City, \\ Kunming, Yunnan, People's Republic of \\ China; ${ }^{2}$ Epidemiology Unit, Faculty of \\ Medicine, Prince of Songkla University, \\ Hat Yai, Songkhla, Thailand; ${ }^{3}$ Department \\ of Preventive Medicine, School of Public \\ Health and Management, Hubei \\ University of Medicine, Shiyan, Hubei, \\ People's Republic of China
}

*These authors contributed equally to this work
This article was published in the following Dove Press journal:

Cancer Management and Research

Background: Data concerning adherence to hepatocellular carcinoma (HCC) surveillance among chronic liver disease (CLD) patients at high risk of developing HCC in China are limited. We aimed to examine the relationship between HCC-related knowledge dimensions and adherence to HCC surveillance procedures among chronic liver disease patients at high risk of developing HCC and to identify potential barriers.

Methods: A total of 380 patients with chronic liver disease at high risk of developing HCC were recruited between May and August 2018 to complete a survey during the first week of their first hospitalization at the Third People's Hospital of Kunming in China. We followed up each patient up to 7 months by telephone to confirm whether the patient returned to complete investigations for HCC surveillance. Patient's socio-demographic characteristics, HCC-related knowledge, and perceived barriers to HCC surveillance were measured using a structured questionnaire during their hospitalization. Factor analysis was performed on the knowledge questions to reduce the dimensions. Univariate and multivariate analyses were performed to examine the association between dimensions of HCC-related knowledge and patients' adherence to HCC surveillance.

Results: A total of 327 eligible patients had been successfully contacted in the follow-up phase. Only a quarter of patients completed HCC surveillance within 7 months after their first admission to hospital. High costs and perceived poor test efficacy were the two major barriers for HCC surveillance. Three common factors were derived from the factor analysis of HCCrelated knowledge, namely, "Surveillance", "Lifestyle", and 'Prognosis'. Knowledge of HCC surveillance and lifestyle but not prognosis had an influence on adherence to HCC surveillance. Patients with better surveillance and lifestyle knowledge domain had better adherence to HCC surveillance.

Conclusion: Adherence to HCC surveillance procedures is low in the study area. Closing the gap in HCC-related knowledge, particularly regarding surveillance and lifestyle, may help to increase adherence rates.

Keywords: HCC surveillance, adherence, knowledge, barriers

\section{Introduction}

Approximately 782,000 new liver cancer cases are diagnosed globally each year, nearly half of which are in China. ${ }^{1,2}$ The prognosis for liver cancer is very poor with an overall mortality rate of $95 \%^{3}$ and a five-year relative survival rate of $10.1 \%$ in China. ${ }^{4}$ Despite recent advances in the treatment of hepatocellular
Correspondence: Virasakdi

Chongsuvivatwong

Email cvirasak@medicine.psu.ac.th 
carcinoma (HCC), which enable patients with early-stage HCC achieve better outcomes, an unacceptable proportion of Chinese patients with symptomatic late-stage diseases remains. ${ }^{5}$ HCC surveillance is a clinical continuous monitoring process for disease occurrence. Several studies have shown that HCC surveillance may detect HCC at an early stage which allows the curative treatment options available, such as tumor ablation, surgical resection, and liver transplantation, thus reducing mortality and improving prognosis. ${ }^{6,7}$ In order to reduce the burden of liver cancer, the Chinese Ministry of Health developed guidelines recommending the practice of surveillance for early cancer detection, including the application of a diagnostic test in clinics by B-scan ultrasonography and serum alphafetoprotein (AFP) together as a panel of tumor markers for surveillance in high-risk patients who are predisposed to develop HCC. $6,8,9$

Adherence to HCC surveillance, as one of the items for chronic liver disease management, is critical to achieving improved health outcomes, quality of life, and cost-effective health care. ${ }^{10}$ The ultimate goal of the management of chronic liver disease is the prevention of advanced liver disease, including both cirrhosis and liver cancer. However, the average adherence rate of patients with chronic diseases is only $50 \% .^{11,12}$ Non-adherence is a serious challenge to liver disease management.

According to the present Chinese medical system, patients are encouraged to seek treatment at community health centers to overcome treatment availability and affordability barriers by giving relatively high rates of reimbursement and increasing public health funds. ${ }^{13,14}$ However, some barriers, such as help-seeking, prevention of transmission, and adherence to treatment, still remain that have a negative impact on patients receiving medical services. Previous studies on cancer surveillance have applied models of behavior change theory and demonstrated that poor knowledge, perceived barriers to surveillance, and negative attitudes may be associated with lower adherence rates. ${ }^{15,16}$ Understanding the lack of knowledge related to $\mathrm{HCC}$ and existing barriers of patients is therefore valuable for finding effective approaches that can be implemented to promote HCC surveillance.

Although a few studies on high-risk patients have reported their knowledge and barriers for HCC screening, the participation rate of HCC surveillance among high-risk patients in clinical practice in Yunnan province is unknown. Moreover, identifying the specific aspects of HCC-related knowledge is of practical significance for improving adherence to HCC surveillance. Therefore, this study aimed to investigate adherence to HCC surveillance among high-risk chronic liver disease patients, examine their HCC-related knowledge, and identify their barriers to the surveillance program.

\section{Methods}

\section{Study Design, Study Setting, and Patient Selection}

This prospective cohort study was conducted between May 2018 and February 2019 at the Third People's Hospital of Kunming in China. This hospital cares for a large proportion of liver disease patients in the area.

The procedure of patients selection is presented in Supplemental Figure 1. Two experienced hepatologists verified the diagnosis based on a combination of factors including clinical characteristics, biochemical parameters, and imaging examinations of each chronic liver disease inpatient. The same two hepatologists also confirmed whether the patients' ICD-10-CM (International Classification of Diseases, 10th revision, Clinical Modification) diagnoses were entered correctly, and then used the ICD-10-CM diagnostic and procedural codes to identify chronic liver disease patients at high risk of developing HCC. High risk was defined as patients with hepatitis $\mathrm{B}$ virus (HBV) infection, hepatitis $\mathrm{C}$ virus (HCV) infection, $\mathrm{HBV}$ and $\mathrm{HCV}$ co-infection, alcoholic liver disease (ALD), nonalcoholic fatty liver disease (NAFLD), and liver cirrhosis of all causes. Patients were excluded if they had hepatocellular carcinoma, endstage renal failure, or end-stage heart failure, if they were grade 3-4 encephalopathy, or if they had received a liver transplant.

All eligible patients were consecutively recruited after being admitted to the Liver Unit of the study hospital. Routine laboratory examinations including complete blood count, coagulation function, serum biochemical tests (liver function, renal function, electrolytes, and AFP) and imaging examinations (B-scan ultrasonography) were performed on admission. Patients would have contrast-enhanced computed tomography or contrast-enhanced magnetic resonance imaging performed if the result of ultrasound was inconclusive. All patients were asked to complete the survey questionnaire during the first week of their first hospitalization for the investigation of their disease. After patients finished the three sections of the questionnaire, we explained to them the benefits of HCC surveillance and the advantages of adherence. According to the diagnostic and treatment 
procedures of the hospital, every patient was advised to return for HCC surveillance every 6 months after discharge. Information about the next appointment was sent to all patients at discharge. This included the address of the appointment place (our hospital liver unit or a community health center), the appointment time (exactly 6 months after discharge), and procedures that would be done (ultrasound and blood test to measure the AFP level).

\section{Data Collection}

The questionnaire of the survey was divided into three sections: (i) socio-demographic characteristics, (ii) HCCrelated knowledge, and (iii) potential barriers to $\mathrm{HCC}$ surveillance. Socio-demographic characteristics included sex, age, ethnicity, marital status, occupation, educational level, personal monthly income, and type of medical insurance. Relevant selection of variables is demonstrated in Figure 1. The selection was guided by a theoretical model of patient behavior for HCC surveillance based on the Health Behavior Framework. ${ }^{17}$

The HCC-related knowledge section was modified from previous studies ${ }^{18-20}$ under consultation with six liver disease experts. This section contained 13 statements each with three choices: "aware", "uncertain", and "unaware" (Table 1).

The third section of the questionnaire assessed selfreported barriers to HCC surveillance and their concern on the procedures at community health centers. Questions were adapted from earlier validated surveys. ${ }^{18,20,21}$ After pretesting the questionnaire on 20 patients, necessary modifications were made. Finally, 10 items of possible barriers were finalized: financial issue, accuracy of the surveillance, inadequate information on the place to receive tests as part of the surveillance procedure, the necessity of surveillance, appointment difficulties, time commitment, transportation, lack of a specialist, fear of finding cancer, and fear of pain. According to the results of the pilot study, a slightly modified list of options for the barriers was given for patients to choose from for general hospitals and community medical centers. The patients were asked to choose the important barriers and rank these by their perceived importance to them. We collected the questionnaires, which assessed self-reported barriers to $\mathrm{HCC}$ surveillance and their concerns on the procedures at community health centers, during their first hospitalization.

Laboratory test results were extracted from the hospital information system. The etiology of liver disease was

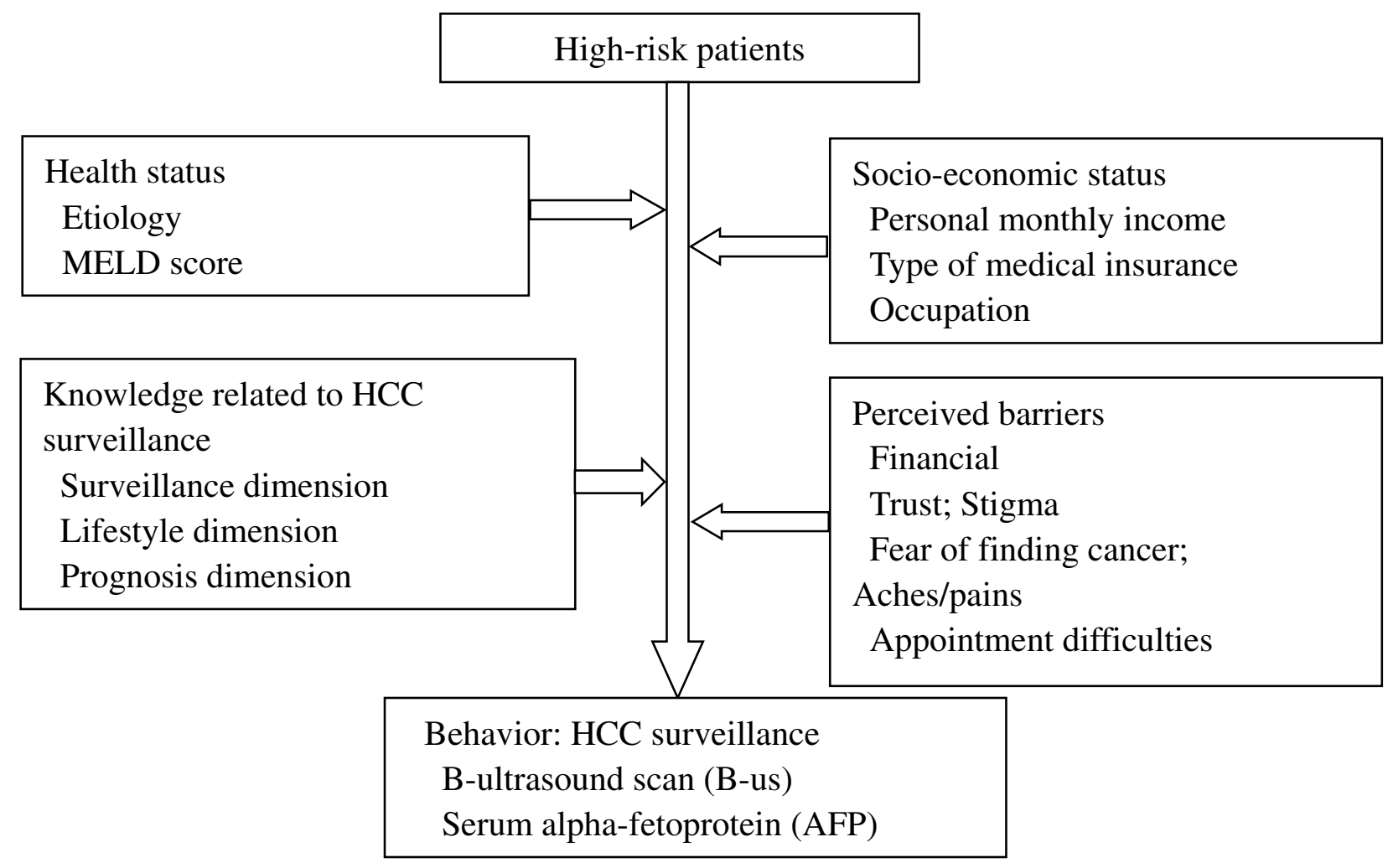

Figure I Model of patient behavior for HCC surveillance. 
Table I Distribution of Knowledge Questions and Their Association with Factor Loadings from Factor Analysis

\begin{tabular}{|c|c|c|c|c|c|c|}
\hline \multirow[t]{2}{*}{ Question } & \multicolumn{3}{|c|}{ Percentage of Distribution (\%) } & \multicolumn{3}{|c|}{ Factor Loading } \\
\hline & Aware & Uncertain & Unaware & Surveillance & Lifestyle & Prognosis \\
\hline QI Patients with cirrhosis are at high risk for developing HCC & 48.3 & 22.3 & 29.4 & 0.332 & 0.568 & 0.206 \\
\hline Q2 Eating a healthy diet does not sufficiently lower the all of $\mathrm{HCC}$ risk & 28.1 & 41.9 & 30.0 & 0.130 & 0.544 & 0.280 \\
\hline Q3 Eating a healthy diet can not preclude a need for surveillance & 46.2 & 22.0 & 31.8 & 0.337 & 0.741 & 0.152 \\
\hline Q4 Lack of abdominal pain does not preclude need for HCC surveillance & 31.5 & 30.9 & 37.6 & 0.406 & 0.630 & 0.101 \\
\hline $\begin{array}{l}\text { Q5 Patients with two consecutive normal surveillance ultrasound exams require } \\
\text { continued HCC surveillance }\end{array}$ & 33.3 & 31.8 & 34.9 & 0.656 & 0.380 & 0.220 \\
\hline Q6 Normal physical exam and labs does not preclude need for $\mathrm{HCC}$ surveillance & 36.4 & 28.4 & 35.2 & 0.750 & 0.336 & 0.138 \\
\hline Q7 Ultrasound is the primary recommended modality for HCC surveillance & 29.7 & 35.5 & 34.9 & 0.498 & 0.148 & 0.338 \\
\hline $\begin{array}{l}\text { Q8 Patients with cirrhosis should have HCC surveillance performed at least once } \\
\text { per half-year }\end{array}$ & 37.3 & 27.8 & 34.9 & 0.622 & 0.329 & 0.162 \\
\hline Q9 Surveillance does not prevent development of HCC & 42.2 & 26.6 & 31.2 & 0.408 & 0.365 & 0.542 \\
\hline QI0 Surveillance does not preclude diagnosis with advanced $\mathrm{HCC}$ & 31.8 & 35.5 & 32.7 & 0.513 & 0.347 & 0.449 \\
\hline QII HCC can be cured if diagnosed at an early stage & 27.8 & 41.6 & 30.6 & 0.338 & 0.156 & 0.413 \\
\hline QI2 Patients with HCC will die without cancer treatment & 66.4 & 17.4 & 16.2 & 0.057 & 0.050 & 0.589 \\
\hline QI3 HCC does not spontaneously resolve without cancer treatment & 55.0 & 20.5 & 24.5 & 0.213 & 0.283 & 0.684 \\
\hline
\end{tabular}

Abbreviation: HCC, hepatocellular carcinoma.

determined from the medical record and classified as hepatitis B virus (HBV), hepatitis $\mathrm{C}$ virus (HCV), and others. The Model for End-Stage Liver Disease (MELD) ${ }^{22}$ score was calculated within 48 hours after admission by applying the standard formula indicating the degree of liver disease severity.

We followed up with each patient between 6 and 7 months after discharge with a telephone call. If there was no response after three attempts, we called one of their relatives. Whether or not the patient returned for surveillance is our primary outcome of interest. The place where they returned for surveillance was also recorded. There are two procedures for HCC surveillance: alpha-fetoprotein (AFP) test and B-scan ultrasonography. If the patient could not clearly mention which procedure they had, we validated the information from the hospital and community health center records.

\section{Data Management and Analysis Analysis of HCC-Related Knowledge}

To reduce the number of dimensions of the HCC-related knowledge, exploratory factor analysis was conducted on the questionnaire items. We used a parallel analysis technique $^{23}$ in combination with a scree plot to determine the ideal number of factors and orthogonal rotation to optimize the configuration of factors. We used factor loadings with an absolute value greater than 0.4 , which explained 53\% of the variance in the variables. The Kaiser-Meyer-Olkin measure of sampling adequacy was computed to verify the adequacy of sample size and the internal consistency of the scale was measured with Cronbach's alpha (0.90). Three dimensions of HCC-related knowledge were obtained and labeled "Surveillance", "Lifestyle" and "Prognosis".

\section{Analysis of Patient-Reported Barriers}

We calculated the percentage of primary barriers and used a heat map to display the distribution.

\section{Analysis of Adherence to HCC Surveillance Procedures}

The B-scan ultrasonography may be replaced with contrast-enhanced computed tomography or contrastenhanced magnetic resonance imaging for surveillance purposes in certain cases. HCC surveillance adherence was taken as an ordinal outcome with three levels: both procedures done, either procedure done, and no procedure done.

\section{Hypothesis Testing and Modeling}

Our main hypothesis was that patient's HCC-related knowledge would be associated with their level of adherence to HCC surveillance. We used the $\chi^{2}$ test or Fisher's exact test for categorical data and the Kruskal-Wallis test for numerical data to examine univariate relationships among groups. An ordinal logistic regression model was then fitted to examine the independent association between dimensions of HCC-related knowledge and patients' adherence to HCC surveillance adjusting for other potential confounders. 
Table 2 Patient's Adherence to HCC Surveillance by Clinical and Social Characteristics $(\mathrm{N}=327)$

\begin{tabular}{|c|c|c|c|c|c|}
\hline \multirow[t]{2}{*}{ Variables } & \multirow[t]{2}{*}{ n (\%) } & \multicolumn{3}{|c|}{ HCC Surveillance Tests (AFP \& B-Scan Ultrasound) } & \multirow[t]{2}{*}{$P$ value } \\
\hline & & Neither (242) & Partial (24) & Both (6I) & \\
\hline Sex & & & & & 0.037 \\
\hline Female & $115(35.2)$ & $89(36.8)$ & $12(50.0)$ & $14(22.9)$ & \\
\hline Male & $212(64.8)$ & $153(63.2)$ & $12(50.0)$ & $47(77.1)$ & \\
\hline Age group (years) & & & & & 0.261 \\
\hline$<40$ & $98(30.0)$ & $80(33.1)$ & $4(16.7)$ & $14(23.0)$ & \\
\hline $40-59$ & $180(55.0)$ & $127(52.5)$ & $17(70.8)$ & $36(59.0)$ & \\
\hline$\geq 60$ & $49(15.0)$ & $35(14.5)$ & $3(12.5)$ & II (I8.0) & \\
\hline Ethnicity & & & & & 0.713 \\
\hline Han & $274(83.8)$ & 205 (84.7) & $19(79.2)$ & $50(82.0)$ & \\
\hline Minority & $53(16.2)$ & $37(15.3)$ & $5(20.8)$ & II (I8.0) & \\
\hline Marital status & & & & & 0.597 \\
\hline Unmarried & $36(11.0)$ & $29(12.0)$ & $3(12.5)$ & $4(6.6)$ & \\
\hline Married & $283(86.5)$ & $207(85.5)$ & $20(83.3)$ & $56(91.8)$ & \\
\hline Divorced/Widowed & $8(2.4)$ & $6(2.5)$ & I (4.2) & I (I.6) & \\
\hline Occupation & & & & & 0.348 \\
\hline Farmer & $126(38.5)$ & $99(38.8)$ & II (45.8) & $21(34.4)$ & \\
\hline Laborer & $50(15.3)$ & $44(18.2)$ & $0(0.0)$ & $6(9.8)$ & \\
\hline Manager & $29(8.9)$ & $18(7.4)$ & $2(8.3)$ & $9(14.8)$ & \\
\hline Professional & $32(9.8)$ & $20(8.3)$ & $3(12.5)$ & $9(14.8)$ & \\
\hline Student & II (3.4) & $7(2.9)$ & I (4.2) & $3(4.9)$ & \\
\hline Retired & $21(6.4)$ & $15(6.2)$ & $2(8.3)$ & $4(6.6)$ & \\
\hline Others & $58(17.7)$ & $24(18.2)$ & $5(20.8)$ & $9(14.8)$ & \\
\hline Educational level & & & & & 0.091 \\
\hline Illiterate & $24(7.3)$ & $22(9.1)$ & I (4.2) & I (I.6) & \\
\hline Primary school & $68(20.8)$ & $55(22.7)$ & $4(16.7)$ & $9(14.8)$ & \\
\hline Middle school & $118(36.1)$ & $89(36.8)$ & $8(33.3)$ & $21(34.4)$ & \\
\hline High school and above & $117(35.8)$ & $76(31.4)$ & II (45.8) & $30(49.2)$ & \\
\hline Personal monthly income & & & & & 0.406 \\
\hline$<800$ & $86(26.3)$ & $66(27.3)$ & $8(33.3)$ & $12(19.7)$ & \\
\hline $800-3000$ & $91(27.8)$ & $70(28.9)$ & $7(29.2)$ & $14(23.0)$ & \\
\hline $3000-6000$ & $109(33.3)$ & $80(33.1)$ & $6(25.0)$ & $23(37.7)$ & \\
\hline$>6000$ & $4 \mid(12.5)$ & $26(10.7)$ & $3(12.5)$ & $12(19.7)$ & \\
\hline Type of insurance & & & & & 0.089 \\
\hline No insurance & $14(4.3)$ & $13(5.4)$ & $0(0.0)$ & $\mathrm{I}(\mathrm{I} .6)$ & \\
\hline NRCMS & $166(50.8)$ & $129(53.3)$ & $13(54.2)$ & $24(39.4)$ & \\
\hline URBMI & $64(19.6)$ & $45(18.6)$ & $7(29.2)$ & $12(19.7)$ & \\
\hline UEBMI & $83(25.4)$ & $55(22.7)$ & $4(16.7)$ & $24(39.3)$ & \\
\hline Cause of disease & & & & & 0.869 \\
\hline HBV & $220(67.3)$ & $162(66.9)$ & $18(75.0)$ & $40(65.6)$ & \\
\hline $\mathrm{HCV}$ & $52(15.9)$ & $38(15.7)$ & $4(16.7)$ & $10(16.4)$ & \\
\hline Others & $55(16.8)$ & $42(17.4)$ & $2(8.3)$ & II (I8.0) & \\
\hline MELD & & & & & 0.927 \\
\hline$<14$ & $275(84.1)$ & 203 (83.9) & 22 (91.7) & $50(82.0)$ & \\
\hline $14-22$ & $4 \mid(12.5)$ & $30(12.4)$ & $2(8.3)$ & $9(14.8)$ & \\
\hline$>22$ & II (3.4) & $9(3.7)$ & $0(0.0)$ & $2(3.3)$ & \\
\hline
\end{tabular}


Table 2 (Continued).

\begin{tabular}{|l|l|l|l|l|l|}
\hline Variables & $\mathbf{n}(\%)$ & \multicolumn{2}{l|}{ HCC Surveillance Tests (AFP \& B-Scan Ultrasound) } \\
\cline { 3 - 6 } & & Neither (242) & Partial (24) & Both (6I) \\
\hline $\begin{array}{l}\text { Surveillance } \\
\text { Median (IQR) }\end{array}$ & $-0.01(-0.73,0.80)$ & $-0.19(-0.80,0.44)$ & $0.23(0.04,0.66)$ & $0.87(0.01,1.04)$ & $<0.001$ \\
\hline $\begin{array}{l}\text { Lifestyle } \\
\text { Median (IQR) }\end{array}$ & $0.06(-0.82,0.75)$ & $-0.07(-0.93,0.64)$ & $0.36(-0.94,0.84)$ & $0.57(0.13,0.84)$ \\
\hline $\begin{array}{l}\text { Prognosis } \\
\text { Median (IQR) }\end{array}$ & $0.10(-0.56,0.65)$ & $0.05(-0.70,0.60)$ & $0.19(-0.54,0.58)$ & $0.42(-0.35,0.74)$ \\
\hline
\end{tabular}

Abbreviations: HCC, hepatocellular carcinoma; HBV, hepatitis B virus; HCV, hepatitis C virus; IQR, inter-quartile range.

All data were computerized using EpiData. All statistical analyses and data visualizations were performed using $\mathrm{R}$ version $3.5 .1 .^{24}$

\section{Ethical Consideration}

The study protocol was approved by the Ethical Committee of the Faculty of Medicine, Prince of Songkla University (REC 61-048-18-1) and the Third People's Hospital of Kunming (Ref: 2017082860). It was conducted in accordance with the Declaration of Helsinki. The patients' personal information such as name and identification number were encrypted before use. Written informed consent was provided by each patient before being interviewed.

\section{Results}

\section{Patients' Characteristics}

A total of 380 eligible patients were recruited between May and July 2018. We followed up each patient between 6 and 7 months after discharge with a telephone call and 327 eligible patients could be contacted and agreed to participate in the study. Reasons for exclusion were inability to contact the patient $(n=29)$, patient refusal $(n=5)$, ultrasound done for diagnosing other conditions $(\mathrm{n}=2)$, admission to hospital $(n=4)$ and death $(n=13)$. A total of 85 patients $(26.0 \%)$ had procedures done as part of the HCC surveillance, 61 having both AFP test and B-scan ultrasound, and 24 having either test. In total, 69 and 16 patients returned to the hospital and a community health center, respectively.

Table 2 presents the demographic and clinical characteristics of the 327 patients. The median age at diagnosis was 46.0 years, with the majority being male (64.8\%). Most patients were of Han ethnicity (83.8\%). The types of medical insurance of participants were NRCMS (New
Rural Cooperative Medical System) (50.8\%), UEBMI (Urban Employee Basic Medical Insurance) (25.4\%), URBMI (Urban Residents' Basic Medical Insurance) (19.6\%). About $36.1 \%$ had a middle school level of education and $35.8 \%$ had completed high school. The most common etiologies of chronic liver disease were HBV infection (67.3\%), HCV infection (15.9\%), and other diseases $(16.8 \%)$ which included alcohol-induced hepatitis, NASH/cryptogenic cirrhosis, and autoimmune diseases. The median personal monthly patient income was 2500 yuan. There were 106 patients $(32.4 \%)$ with cirrhosis. Most of the patients' MELD score was less than 14 indicating a moderate severity of chronic liver disease.

\section{Patients' Knowledge of HCC Surveillance}

Table 1 summarizes patients' HCC-related knowledge. About $53 \%$ of the total variance could be explained in three dimensions. The factor loadings for each of these dimensions are presented in the three right-most columns of Table 1. Factor 1 was labeled "Surveillance knowledge" because the content of the questions was related to HCC surveillance effectiveness. Factor 2 was labeled "Lifestyle knowledge" as the questions focused on HCC risk of daily lifestyle. Factor 3 was labeled "Prognosis knowledge" as the questions concerned HCC treatment and prognosis.

\section{Univariate Analysis}

Table 2 shows the univariate association between adherence to HCC surveillance and socio-demographic and clinical characteristics and HCC-related knowledge. Factors significantly associated with adherence included sex, surveillance knowledge and lifestyle knowledge. Age, ethnicity, marital status, occupation, education level, type of insurance, personal monthly income, cause of disease, MELD score and prognosis knowledge were not 
significant factors for adherence to HCC surveillance in the univariate analysis.

\section{Multivariable Analysis}

Table 3 shows the factors associated with adherence to HCC surveillance on multivariate analysis. The only significant factors were surveillance and lifestyle knowledge; patients with better surveillance knowledge $(\mathrm{OR}=2.83,95 \% \mathrm{CI}$ : 1.92-4.27) and lifestyle knowledge (OR=1.46, 95\% CI: 1.02-2.12) had better adherence to HCC surveillance.

\section{Barriers to HCC Surveillance}

Figure 2 summarizes the 10 most common barriers of returning to complete procedures for HCC surveillance stratified by various patient variables. Supplemental Table 1 presents detailed information about patients' perceived barriers to return for HCC surveillance. There were two major barriers: expense and perceived poor test efficacy. These two barriers are shown in the two left-most columns. Red tone indicates a high concentration of the relative frequency while yellow represents low concentration. There was relatively little contrast across categories of sex, age, and ethnicity. However, expense was a more common concern by patients who had a low income, were divorced or widowed, illiterate, and had HCV. Perceived poor test efficacy was a more common concern among patients aged more than 60 , and those who worked as a manager. Fear of finding cancer was a more common concern among patients whose MELD score was more than 22 .

Table 3 Ordinal Logistic Regression of Clinical and Social Factors Predicting Patient's Adherence to HCC Surveillance $(\mathrm{N}=327)$

\begin{tabular}{|c|c|c|c|c|}
\hline Variables & OR & $95 \% \mathrm{Cl}$ & P-value (Wald's Test) & P-value (LR-Test) \\
\hline Sex: Male vs Female & 1.45 & $(0.79,2.72)$ & 0.118 & 0.233 \\
\hline Age group (years): ref. $=<40$ & & & & 0.391 \\
\hline $40-59$ & 1.69 & $(0.80,3.75)$ & 0.091 & \\
\hline$\geq 60$ & 1.68 & $(0.55,5.06)$ & 0.179 & \\
\hline Ethnicity: Minority vs Han & 1.27 & $(0.6 \mathrm{I}, 2.58)$ & 0.260 & 0.523 \\
\hline Marital status: ref.= Unmarried & & & & 0.417 \\
\hline Married & 2.27 & $(0.64,10.90)$ & 0.122 & \\
\hline Divorced/Widowed & 1.50 & $(0.14,14.62)$ & 0.363 & \\
\hline Occupation: ref.= Farmer & & & & 0.218 \\
\hline Laborer & 0.34 & $(0.09,1.08)$ & 0.040 & \\
\hline Manager & 1.14 & $(0.34,3.82)$ & 0.415 & \\
\hline Professional & 0.79 & $(0.2 \mathrm{I}, 2.80)$ & 0.358 & \\
\hline Student & 4.66 & $(0.6 I, 38.74)$ & 0.070 & \\
\hline Retired & 0.51 & $(0.11,2.33)$ & 0.195 & \\
\hline Others & 1.05 & $(0.40,2.72)$ & 0.460 & \\
\hline Personal monthly income: ref. $=<800$ & & & & 0.840 \\
\hline $800-3000$ & 1.00 & $(0.43,2.35)$ & 0.498 & \\
\hline $3000-6000$ & 1.05 & $(0.4 \mathrm{I}, 2.68)$ & 0.464 & \\
\hline$>6000$ & 1.52 & $(0.48,4.81)$ & 0.236 & \\
\hline Type of insurance: ref.= No insurance & & & & 0.132 \\
\hline NRCMS & 4.46 & $(0.72,86.85)$ & 0.089 & \\
\hline URBMI & 3.88 & $(0.61,76.73)$ & 0.114 & \\
\hline UEBMI & 8.17 & $(1.25,164.65)$ & 0.033 & \\
\hline Cause of disease: ref.= HBV & & & & 0.872 \\
\hline $\mathrm{HCV}$ & 1.17 & $(0.5 \mathrm{I}, 2.58)$ & 0.355 & \\
\hline Others & 0.89 & $(0.39,1.92)$ & 0.385 & \\
\hline Surveillance (continuous variable) & 2.83 & $(1.92,4.27)$ & $<0.001$ & $<0.001$ \\
\hline Lifestyle (continuous variable) & 1.46 & $(1.02,2.12)$ & 0.022 & 0.041 \\
\hline Prognosis (continuous variable) & 1.04 & $(0.72,1.52)$ & 0.412 & 0.823 \\
\hline
\end{tabular}

Abbreviations: $\mathrm{OR}$, odds ratio; $\mathrm{Cl}$, confidence interval; ref, reference group; $\mathrm{HBV}$, hepatitis $\mathrm{B}$ virus; $\mathrm{HCV}$, hepatitis $\mathrm{C}$ virus. 

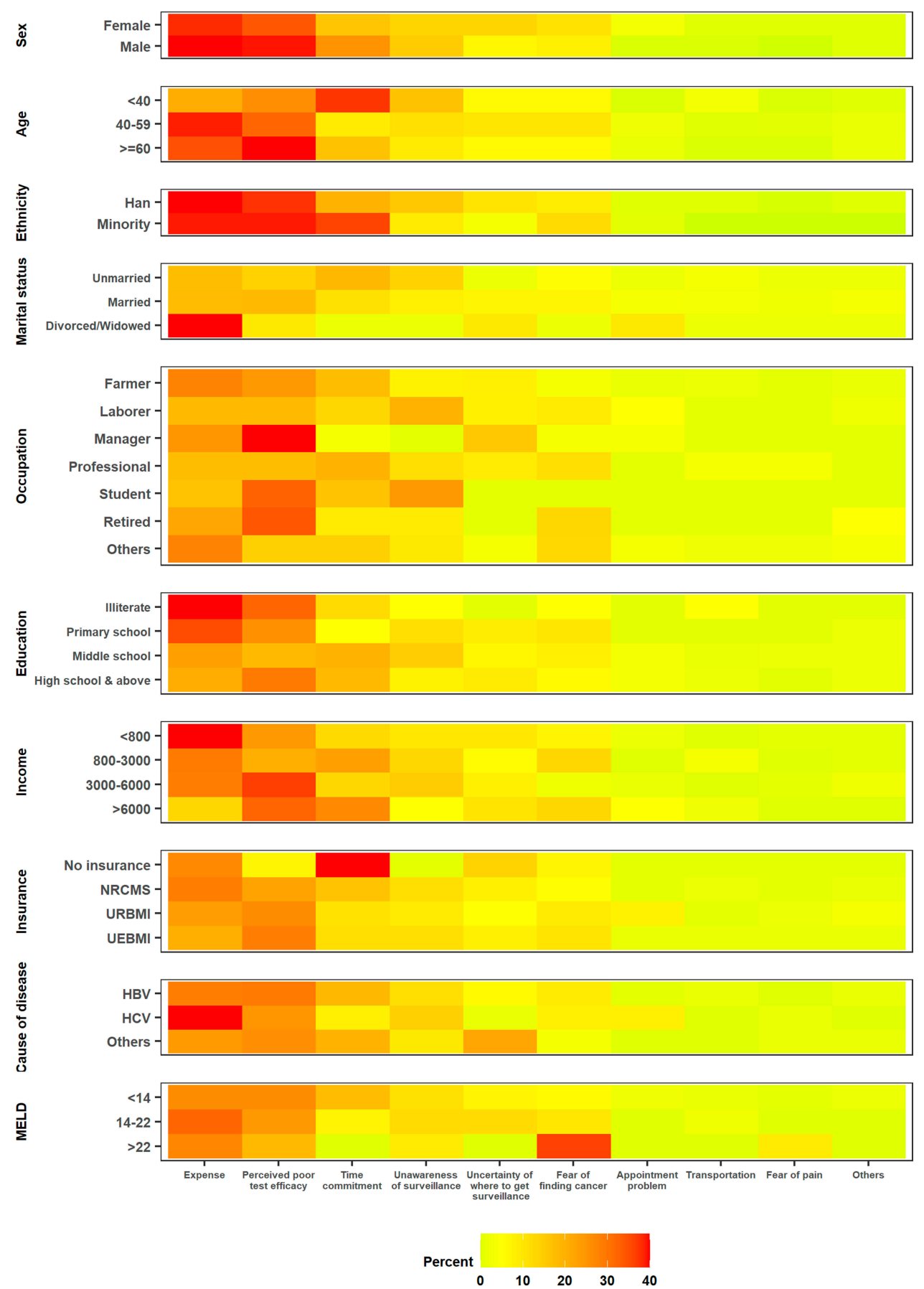

Figure 2 Perceived barriers to return for HCC surveillance.

Figure 3 summarizes the six barriers for community HCC surveillance stratified by various variables. Supplemental Table 2 presents detailed information about reasons not performing HCC Surveillance in community health centers. There was relatively little contrast across categories of sex, age, and cause of disease. However, perceived poor test efficacy was a more common concern among those who were married, retired, more educated, wealthier, and insured under the UEBMI scheme. Fear of finding cancer was more common among patients whose MELD score was more than 22 .

\section{Discussion}

Only one-quarter of patients returned to undergo $\mathrm{HCC}$ surveillance procedures within 7 months of their first hospitalization for chronic liver disease. Of those who did 

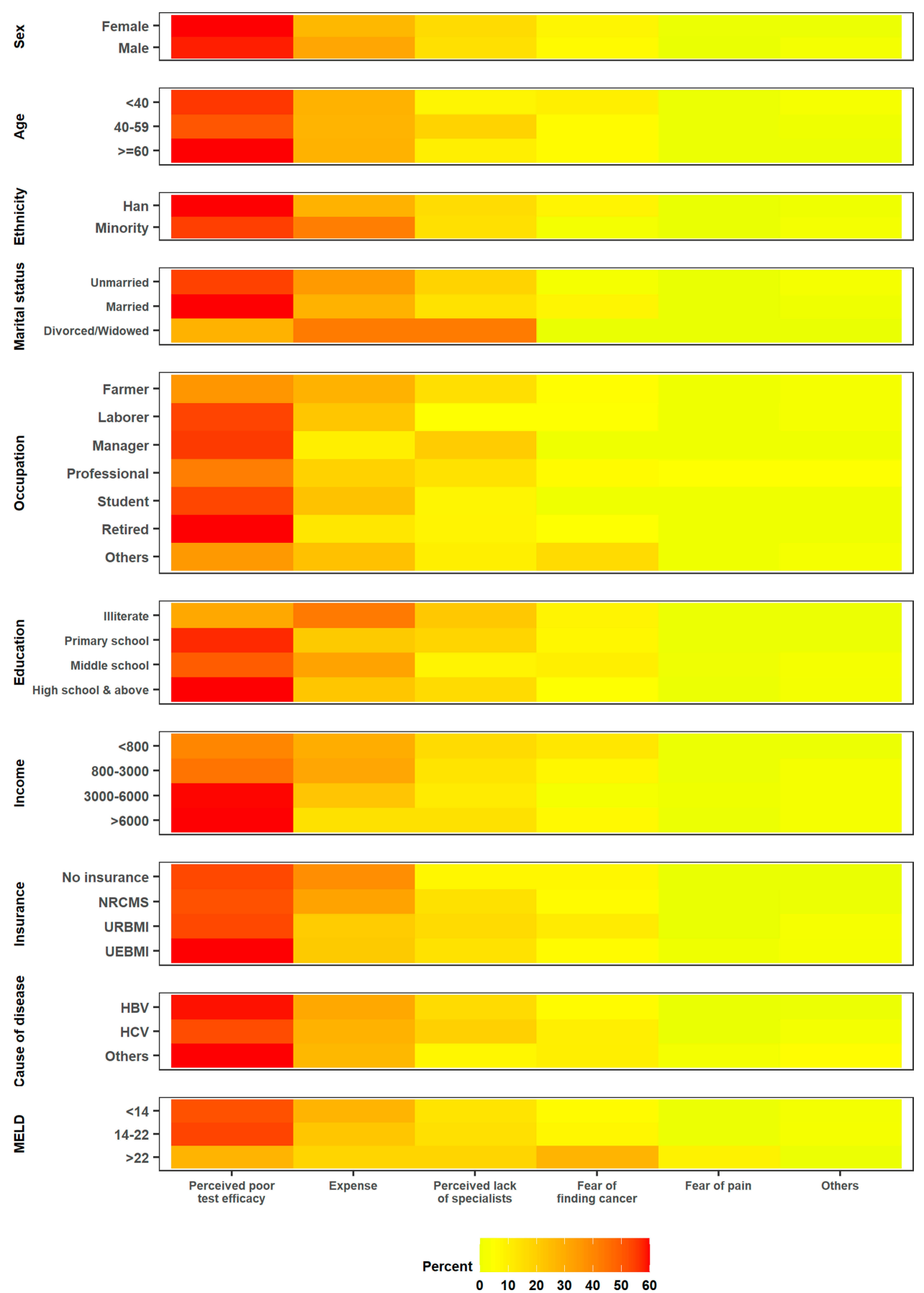

Figure 3 Reasons for not performing HCC surveillance in primary health centers.

return, about $72 \%$ completed all procedures. Three dimensions of HCC-related knowledge were identified, two of which, namely "Surveillance knowledge" and "Lifestyle knowledge", could predict adherence to HCC surveillance. The main barriers of adherence to HCC surveillance reported by patients were cost, trust in efficacy of the tests, and transportation difficulties. In community health centers, trust in test efficacy was the main barrier. A small number of patients reported stigma as one of the barriers.

The adherence practice of HCC surveillance among high-risk chronic liver disease patients in our study was generally lower compared with those of other studies. ${ }^{25}$ Several countries have engaged in nationwide systematic and active use of HCC surveillance programs, ${ }^{26}$ but China 
relies largely on individual acceptance and participation in these programs. ${ }^{27}$

Of all items of the knowledge questions, less than $40 \%$ were correctly answered. This reflected the gap between doctors and high-risk patients' inadequate knowledge. Adherence to HCC surveillance could be predicted by "Surveillance knowledge" and "Lifestyle knowledge" but not "Prognosis knowledge". This result may be explained from the behavior theory: from understanding knowledge, awareness of the need to change, intention to change, to an actual change in behavior. ${ }^{28}$ Knowledge and barriers may also bypass intentions and exert a direct influence on health behaviors. ${ }^{29}$ This suggests that it may be more useful for patients' adherence practice to master specific or direct knowledge of HCC surveillance. High-risk patients should be provided extensive education about the effectiveness of HCC surveillance and HCC risk through daily lifestyles.

Understanding the barriers of adherence to HCC surveillance can help to find ways to overcome them. Patients reported several barriers, including cost, lack of awareness, and transportation difficulties. A quarter of patients rated "Worry about the expense" as the most important barrier. Lack of trust in test efficacy was the most common barrier in community health centers. However, both variables (expense and trust) were not significantly associated with adherence to HCC surveillance.

Social and financial factors such as personal monthly income and insurance were not significant predictors of adherence to HCC surveillance. There is no reimbursement policy for outpatients' examinations ${ }^{27}$ in any type of insurance, which may explain this result.

A fewer number of patients with higher personal monthly income reported "expense" as a major barrier. Although personal monthly income was not significantly associated with adherence to HCC surveillance, many patients did express concern about the cost of the surveillance tests. The explanation of this result has three aspects. First, we asked patients the barriers 6 months before they returned for HCC surveillance. During this period, their financial situation may have changed. Second, in China, a large proportion of rural residents believe that healthcare services are expensive, ${ }^{30}$ thus affordability is a large concern for many. ${ }^{31}$ It is natural for many of us to worry about expenses for any service. Third, the cost of tests may be increased due to other examinations not mentioned in the surveillance program. ${ }^{32}$ Simple reassurance about insurance coverage may be sufficient to allay fears of $\mathrm{HCC}$ surveillance expense and having the schedule of HCC surveillance the same day as return visits can likely mitigate patient issues with time commitment, transportation and so on. In our study, patients whose MELD score was more than 22 reported fear of finding cancer as the main barrier to HCC surveillance. The fact that serious patients showed psychological fear suggests that we should pay more attention to them and provide professional counseling to assist them to cope with this fear.

Trust in test efficacy was the most common barrier for patients in community health centers. This finding is similar to the result of a survey conducted in China. ${ }^{21}$ The routine work of community health centers generally involves caring for patients with high blood pressure, diabetes, heart disease and so on; however, professional treatment provided for patients with chronic liver disease is rare. ${ }^{33}$ This condition can contribute potential trust issues that can affect patient's adherence to HCC surveillance in the community. This highlights the need for additional training to community physicians and specialist supporting mechanisms as well as a practical complete HCC surveillance system to help improve the current practice.

Among all participants from community health centers, two reported stigma as the most important barrier. Patients who feel stigmatized use hiding strategies and will probably experience a higher pressure to seek treatment and therefore risk hindering access to social support and early treatment. ${ }^{34}$ It is particularly imperative to effectively protect patients' privacy to improve the participation rate of HCC surveillance in community health centers.

Our study is subject to some limitations. The data were obtained from a liver disease department in an infectious disease tertiary hospital, predominated by $\mathrm{HBV}$ and $\mathrm{HCV}$ patients. We did not have enough data on liver disease due to other causes such as alcohol and immune chronic liver disease. In addition, most of the eligible patients were young; thus, the generalizability of our results in elderly patients as well as those with other etiologies must be made with caution. Moreover, HCC surveillance for patients with chronic liver disease should be a long-term process, but our study observed only one period and could not fully evaluate the surveillance dynamics of patients.

Our study suggests that HCC surveillance rates are low in our research district. The study revealed some opportunities to increase surveillance rates by closing the gap in HCC-related knowledge, particularly regarding surveillance and lifestyle knowledge. Overall, these findings 
highlight the need for patient communication to increase HCC surveillance adherence rates.

\section{Acknowledgments}

Dr Dai would like to thank the support from the Health Commission of Kunming city (Grant No. 2020-03-08-112). Dr. Jun Zhao received a grant from Hubei University of Medicine (Grant No. 2018QDJZR14).

\section{Disclosure}

The authors report no conflicts of interest in this work.

\section{References}

1. Ferlay J, Soerjomataram I, Dikshit R, et al. Cancer incidence and mortality worldwide: sources, methods and major patterns in GLOBOCAN 2012. Int $J$ Cancer. 2015;136(5):E359-E386. doi:10.1002/ijc. 29210

2. Chen W, Zheng R, Zhang S, et al. Cancer incidence and mortality in China, 2013. Cancer Lett. 2017;401:63-71. doi:10.1016/j. canlet.2017.04.024

3. Omata M, Cheng AL, Kokudo N, et al. Asia-Pacific clinical practice guidelines on the management of hepatocellular carcinoma: a 2017 update. Hepatol Int. 2017;11(4):317-370.

4. Zeng H, Zheng R, Guo Y, et al. Cancer survival in China, 2003-2005: a population-based study. Int $J$ Cancer. 2015;136(8):1921-1930. doi:10.1002/ijc.29227

5. Yang JD, Mannalithara A, Piscitello AJ, et al. Impact of surveillance for hepatocellular carcinoma on survival in patients with compensated cirrhosis. Hepatology. 2018;68(1):78-88. doi:10.1002/ hep. 29594

6. Zhang BH, Yang BH, Tang ZY. Randomized controlled trial of screening for hepatocellular carcinoma. J Cancer Res Clin Oncol. 2004;130(7):417-422. doi:10.1007/s00432-004-0552-0

7. Yang JD, Harmsen WS, Slettedahl SW, et al. Factors that affect risk for hepatocellular carcinoma and effects of surveillance. Clin Gastroenterol Hepatol. 2011;9(7):617-623. doi:10.1016/j. cgh.2011.03.027

8. Zhou J, Sun HC, Wang Z, et al. Guidelines for diagnosis and treatment of primary liver cancer in China (2017 edition). Liver Cancer. 2018;7(3):235-260. doi:10.1159/000488035

9. Wang FS, Fan JG, Zhang Z, Gao B, Wang HY. The global burden of liver disease: the major impact of China. Hepatology. 2014;60 (6):2099-2108. doi:10.1002/hep.27406

10. Viswanathan $\mathrm{M}$, Golin CE, Jones CD, et al. Interventions to improve adherence to self-administered medications for chronic diseases in the United States: a systematic review. Ann Intern Med. 2012;157 (11):785-795. doi:10.7326/0003-4819-157-11-201212040-00538

11. Burkhart PV, Sabate E. Adherence to long-term therapies: evidence for action. J Nurs Scholarship. 2003;35(3):207.

12. Sabaté E. Adherence to Long-Term Therapies: Evidence for Action. World Health Organization; 2003.

13. Barber SL, Yao L. Development and status of health insurance systems in China. Int $J$ Health Plan M. 2011;26(4):339-356. doi: $10.1002 / \mathrm{hpm} .1109$

14. Liang W, Xie J, Fu H, Wu EQ. The role of health economics and outcomes research in health care reform in China. PharmacoEconomics. 2014;32(3):231-234. doi:10.1007/s40273014-0141-2

15. Rakowski W, Breslau ES. Perspectives on behavioral and social science research on cancer screening. Cancer. 2004;101(5 Suppl):1118-1130. doi:10.1002/cncr.20503
16. Klein WM, Bloch M, Hesse BW, et al. Behavioral research in cancer prevention and control: a look to the future. Am J Prev Med. 2014;46 (3):303-311. doi:10.1016/j.amepre.2013.10.004

17. Nguyen TT, Taylor V, Chen MS Jr, Bastani R, Maxwell AE, McPhee SJ. Hepatitis B awareness, knowledge, and screening among Asian Americans. J Cancer Educ. 2007;22(4):266-272. doi:10.1007/ BF03174128

18. Farvardin S, Patel J, Khambaty M, et al. Patient-reported barriers are associated with lower hepatocellular carcinoma surveillance rates in patients with cirrhosis. Hepatology. 2017;65(3):875-884. doi: $10.1002 /$ hep. 28770

19. Sharma P, Saini SD, Kuhn LB, et al. Knowledge of hepatocellular carcinoma screening guidelines and clinical practices among gastroenterologists. Dig Dis Sci. 2011;56(2):569-577. doi:10.1007/s10620010-1453-5

20. Xu K, Watanabe-Galloway S, Rochling FA, et al. Practice, knowledge, and barriers for screening of hepatocellular carcinoma among high-risk chinese patients. Ann Glob Health. 2017;83(2):281-292. doi:10.1016/j.aogh.2017.02.002

21. Yang Y, Yang D. Community health service centers in China, not always trusted by the populations they serve? China Economic Rev. 2009;20(4):620-624. doi:10.1016/j.chieco.2009.04.004

22. Kamath PS, Wiesner RH, Malinchoc M, et al. A model to predict survival in patients with end-stage liver disease. Hepatology. 2001;33 (2):464-470. doi:10.1053/jhep.2001.22172

23. Humphreys LG, Jr RGM. An investigation of the parallel analysis criterion for determining the number of common factors. Multivar Behav Res. 1975;10(2):193-205. doi:10.1207/s15327906mbr1002_5

24. R Core Team. R: A Language and Environment for Statistical Computing. Vienna, Austria: R Foundation for Statistical Computing; 2018. Available from: http://www.R-project.org/. Accessed July 13, 2020.

25. Zhao C, Jin M, Le RH, et al. Mo1505 Meta-Analysis: hepatocellular Carcinoma (HCC) Surveillance Adherence Rate (SAR) in High Risk Patients. Gastroenterology. 2016;150(4):S1134. doi:10.1016/S00165085(16)33827-6

26. Purcell Y, Copin P, Paulatto L, Pommier R, Vilgrain V, Ronot M. Hepatocellular carcinoma surveillance: eastern and Western perspectives. Ultrasonography. 2018;13:154.

27. Hu S, Tang S, Liu Y, Zhao Y, Escobar ML, de Ferranti D. Reform of how health care is paid for in China: challenges and opportunities. Lancet. 2008;372(9652):1846-1853. doi:10.1016/S0140-6736(08)61368-9

28. Glanz K, Bishop DB. The role of behavioral science theory in development and implementation of public health interventions. Annu Rev Public Health. 2010;31(1):399-418. doi:10.1146/annurev. publhealth.012809.103604

29. Bastani R, Glenn BA, Taylor VM, et al. Integrating theory into community interventions to reduce liver cancer disparities: the health behavior framework. Prev Med. 2010;50(1-2):63-67. doi:10.1016/j. ypmed.2009.08.010

30. Yu-lin Q, Jing-wei Z, Can X, Xiao-li X, Hua-yang H. Research of satisfactory degree on urban residents health insurance: tiantai county as an example [J]. Chin J Health Policy. 2009;2.

31. Wang X, He X, Zheng A, Ji X. The effects of China's New Cooperative Medical Scheme on accessibility and affordability of healthcare services: an empirical research in Liaoning Province. BMC Health Serv Res. 2014;14(1):388. doi:10.1186/1472-6963-14388

32. Yip WC, Hsiao WC, Chen W, Hu S, Ma J, Maynard A. Early appraisal of China's huge and complex health-care reforms. Lancet. 2012;379(9818):833-842. doi:10.1016/S0140-6736(11)61880-1

33. Li T, Su S, Zhao Y, et al. Barriers to the prevention and control of hepatitis $\mathrm{b}$ and hepatitis $\mathrm{c}$ in the community of southwestern china: a qualitative research. Int J Environ Res Public Health. 2019;16:2.

34. HassanpourDehkordi A, Mohammadi N, NikbakhatNasrabadi A. Hepatitis-related stigma in chronic patients: A qualitative study. Appl Nurs Res. 2016;29:206-210. doi:10.1016/j.apnr.2015.04.010 


\section{Publish your work in this journal}

Cancer Management and Research is an international, peer-reviewed open access journal focusing on cancer research and the optimal use of preventative and integrated treatment interventions to achieve improved outcomes, enhanced survival and quality of life for the cancer patient.

The manuscript management system is completely online and includes a very quick and fair peer-review system, which is all easy to use. Visit http://www.dovepress.com/testimonials.php to read real quotes from published authors

Submit your manuscript here: https://www.dovepress.com/cancer-management-and-research-journal 